\title{
Identification of Rare Variants from Exome Sequence in a Large Pedigree with Autism
}

\author{
E.E. Marchani ${ }^{a} \quad$ N.H. Chapman ${ }^{a} \quad$ C.Y.K. Cheung ${ }^{\text {b }} \quad$ K. Ankenman ${ }^{c} \quad$ I.B. Stanaway ${ }^{d}$ \\ H.H. Coon ${ }^{\mathrm{e}}$ D. Nickerson ${ }^{d}$ R. Bernier ${ }^{c} \quad$ Z. Brkanac $^{c}$ E.M. Wijsman ${ }^{\mathrm{a}, \mathrm{b}, \mathrm{d}}$

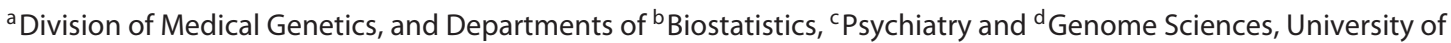 \\ Washington, Seattle, Wash., and ${ }^{~}$ Department of Psychiatry, University of Utah, Salt Lake City, Utah, USA
}

\section{Key Words}

Imputation · Inheritance vector · Linkage analysis ·

Haplotype $\cdot$ MCMC

\begin{abstract}
We carried out analyses with the goal of identifying rare variants in exome sequence data that contribute to disease risk for a complex trait. We analyzed a large, 47-member, multigenerational pedigree with 11 cases of autism spectrum disorder, using genotypes from 3 technologies representing increasing resolution: a multiallelic linkage marker panel, a dense diallelic marker panel, and variants from exome sequencing. Genome-scan marker genotypes were available on most subjects, and exome sequence data was available on 5 subjects. We used genome-scan linkage analysis to identify and prioritize the chromosome 22 region of interest, and to select subjects for exome sequencing. Inheritance vectors (IVs) generated by Markov chain Monte Carlo analysis of multilocus marker data were the foundation of most analyses. Genotype imputation used IVs to determine which sequence variants reside on the haplotype that co-segregates with the autism diagnosis. Together with a rare-allele frequency filter, we identified only one rare variant on the risk haplotype, illustrating the potential of this approach to
\end{abstract}

prioritize variants. The associated gene, MYH9, is biologically unlikely, and we speculate that for this complex trait, the key variants may lie outside the exome.

Copyright $\odot 2013$ S. Karger AG, Basel

\section{Introduction}

Pedigree-based designs have long been a cornerstone of genetic analysis. Methods for linkage analysis in extended human pedigrees were introduced and implemented years before DNA-specific technologies existed to provide genotypes at polymorphic loci [1-3]. However, it was the vast potential of DNA markers [4] and the early success in mapping the Huntington's disease gene [5] that solidified the importance of pedigree-based designs for disease-gene identification. The combination of genome scans for linkage and increasingly informative DNA markers led to the identification of approximately 4,500 disease genes [6], with many of these diseases adequately modeled with a relatively simple mode of inheritance. Identification of genes in which mutations cause extreme cases of otherwise complex diseases provides additional evidence of success, such as early-onset breast cancer [7] and Alzheimer's disease [8, 9]. It has been far

\section{KARGER}

E-Mail karger@karger.com

www.karger.com/hhe
(C) 2013 S. Karger AG, Basel

0001-5652/12/0744-0153\$38.00/0
Ellen M. Wijsman

University of Washington

4333 Brooklyn Ave NE, Box 359460

Seattle, WA 98195-9460 (USA)

E-Mail Wijsman@u.washington.edu 
more challenging to identify genes contributing to complex traits, including late-onset breast cancer and $\mathrm{Alz}$ heimer's disease, as well as others for which Mendelian forms have not been found, such as autism and schizophrenia.

Assumptions about the genetic architecture of complex traits, and approaches for pursuing their underlying genetic basis, have changed over time. Analysis of pedigrees is well-suited for the study of disorders caused by rare variants; linkage analysis is less powerful when the underlying risk variants are common [10]. When linkage analyses struggled to identify genes underlying complex traits, the hypothesis that common variants are responsible for common traits [11] was proposed, favoring study designs comparing unrelated samples over pedigreebased designs [10]. This led to large numbers of genomewide association studies (GWAS) testing association between phenotype and densely spaced markers, with more than 1,340 publications since 2005 reporting at least one statistically significant association (August 2012; www. genome.gov/gwastudies). However, convincing identification of the underlying risk variants is still rare $[12,13]$, and only a small fraction of disease risk is so far explained by GWAS results [14]. Therefore, there has been a recent re-evaluation of the importance of rare variants as a component of the genetic architecture of complex traits [14]. This has renewed interest in analytical designs in which pedigrees are carefully phenotyped and selected for aggregation of unusual phenotypes $[15,16]$. Large pedigrees also offer both greater power and resolution to localize a particular variant when compared with smaller pedigrees [17]. This design has been shown in simulation studies to have strong advantages over GWAS for identifying rare variants with relatively high penetrance $[18,19]$. The few genetic variants with convincing evidence for involvement in complex traits are mostly rare and were identified in large pedigrees [20-22], further supporting this design. It is worth noting that there are also many published genome scans of complex traits with strong evidence for linkage that have not yet yielded convincing risk variants. These are prime targets for further research/study [16].

Technical challenges posed an additional obstacle to identifying the genetic basis of complex traits. Until recently, it was difficult and/or costly to perform the extensive sequencing and statistical analysis [16] necessary for comprehensive evaluation of the relatively large regions identified by linkage scans. Without these thorough analyses, it is difficult to determine whether or not a region with strong support for linkage in fact harbors risk variants. Fortunately, these constraints are being removed by advances in sequencing techniques [23-25], analytical methods $[15,16,26]$, and computational speed and cost efficiency. However, 2 important practical constraints remain. First, sequencing costs remain high, even though they have dropped precipitously in the last couple of years. This limits the number of subjects that can be sequenced for budgetary reasons. Second, because highthroughput sequencing methods require relatively large amounts of high-quality DNA, this can be particularly limiting for older studies or for those that involve young children, where DNA sample quantity may be low. These constraints suggest that there is value in methods that can effectively combine the information from standard genotyping on most family members and newer sequence data collected in a subset of family members.

We show that it is possible to address constraints of both cost and sample availability by imputing, rather than directly measuring, sequence variants on members of a large, multigenerational pedigree with multiple cases of autism. We combine direct measurement of exome variants from high-throughput sequencing, available on only a small number of subjects, with existing genome-scan marker data available on most of their relatives. For the current discussion, we focus attention on a region with positive evidence of linkage. The genome-scan markers provide information about the inheritance of chromosomal segments, as well as the approximate locations of recombination events. This evidence guides our selection of subjects for sequencing, and provides a framework from which discovered sequence variants can be imputed into many of their relatives. The success of this approach in narrowing the field to a very small number of plausible variants illustrates the potential of our approach.

\section{Methods}

\section{Overview}

Our strategy was to use linkage analysis of a large pedigree segregating autism to identify a genomic region of interest (ROI), and then combine patterns of identity-by-descent (IBD) sharing with standard variant filters to identify genetic variants co-segregating with disease. Our genome scan of a 10-cM microsatellite (STR) panel identified the genomic region with strongest evidence of linkage, which we followed up with denser markers including single nucleotide polymorphisms (SNPs) to more precisely define its boundaries. For this ROI, we identified a fully informative STR (highly polymorphic in our pedigree, such that the founder chromosome of origin is clear in all individuals) with one allele tagging the primary haplotype segregating with the trait, and used this information to select 5 subjects for exome sequence data collection. We then coupled this sequence data with the existing marker data to impute exome variants into additional subjects. Our goals were: 
first, to illustrate the potential information provided by a large pedigree regarding imputation; second, to evaluate factors affecting the imputation rate, and third, to explore what imputation could add in this particular autism pedigree. Overall, this experiment is an example of using a filter based on linkage evidence, rather than predictions about functional effect, to reduce the number of variants under consideration.

\section{Sample and Data}

The large pedigree we use as an example originally came to our attention through two independently ascertained nuclear families, each with a sib-pair affected by autism spectrum disorder (ASD). Affectation status was defined using criteria described in detail elsewhere [27]. The relationship between these two families was quickly identified. During long-term follow-up, additional children were born in both the original two nuclear families as well as to siblings and cousins of the original parents, with some developing ASD. Figure 1 shows a schematic representation of the pedigree, with unaffected individuals omitted if they had no offspring. Gender and precise numbers of affected children are obscured in this highly unusual pedigree to protect confidentiality: to our knowledge, this pedigree has more affected subjects than any other pedigree previously described in publications as part of a genetic analysis of ASD [28-31]. The pedigree as analyzed includes 47 subjects, with 11 founders and 36 non-founders in 5 generations. DNA was available for all but only 1 pair of deceased founders (generation I). All 9 other founders were married-in spouses. Six nuclear families with 1-4 affected subjects and 2-6 full siblings per family in 2 major pedigree branches contain all 11 affected subjects. As is observed in the few other studies including large families with ASD [28, 32-34], the early generations contain no affected individuals. Existence of multiple cases in multiple branches of the pedigree strongly suggests a reduced-penetrant autosomal dominant mode of inheritance, although the complex nature of ASD also means that there is a strong possibility of more than one cause of the phenotype in this pedigree.

Data was integrated from 3 genotyping platforms, representing increasing marker density: genome-scan STRs, dense SNPs, and exome sequence variants. Prevention Genetics provided STR genotypes for the Marshfield screening set 16, while SNPs (Illumina OmniExpress 12v1) and exome sequence data $[23,24,35,36]$ were provided by the University of Washington Genome Sciences Genomic Resource Center. Genotypes from the ABI STR panel were previously collected for a subset of 18 individuals, before additional subjects with ASD were identified [27]. Capture of the exome and surrounding regions was done using the NimbleGen in-solution SeqCap EZ Exome Library v2.0, with $36.5 \mathrm{Mb}$ being the size of the target regions, resulting in probes covering $44.1 \mathrm{Mb}$. Pairedend sequencing was done with $50 \mathrm{bp}$ reads, with a mean read depth of 131.6 , and with $92.8 \%$ of the target covered with more than 8 reads. Targeted resequencing of the linkage regions was determined to be too costly, regarding both time and budget, for only 6 samples. We chose to sequence exomes rather than genomes because of ease of interpretation and lower cost to achieve high read depth (at the time of the decision). This exome strategy has been successful in other analyses of autism, see for example [37]. All 45 sampled subjects were genotyped for STRs and SNPs, representing all but the first-generation founder couple. All STR and SNP genotypes underwent standard quality-control procedures, including the exclusion of SNP loci with evidence of Mendelian incon-

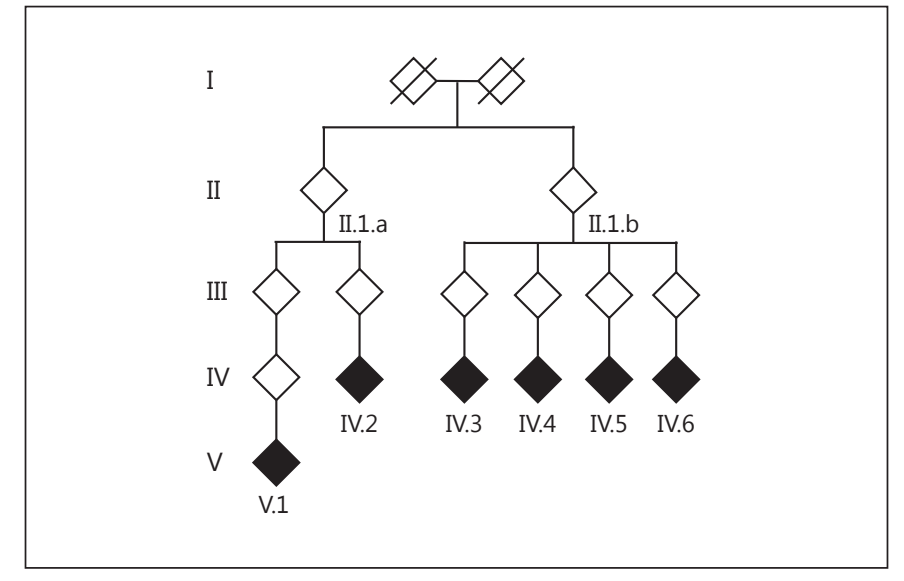

Fig. 1. Schematic representation of the pedigree affected by autism. Symbols illustrate relationships between the 6 affected sibships, represented by filled diamonds in the bottom generations. Only unaffected subjects required to illustrate the relationships between the affected sibships are drawn, all others are excluded from the diagram.

sistencies and high frequency of missing genotypes [38]. SNP genotyping failed on 3 subjects. Prior to the completion of SNP genotyping, we used inferred IBD sharing estimated from the genome scan marker data, described in the Results section, to select 5 subjects for exome sequencing. Subjects were selected for sequencing to maximize phase information regarding the risk haplotype, not necessarily to maximize the number of variants imputed throughout the pedigree.

Analysis Methods

Linkage Analyses

Linkage analyses were carried out with 2 parametric trait models. One model assumed a reduced-penetrant dominant model with allowance for sporadic cases. Penetrances for the high-risk and low-risk genotypes were arbitrarily assumed to be 0.5 and 0.01 , respectively, with a high-risk allele frequency of 0.01 . Phenotypes were coded as affected (11 subjects), unaffected (32 subjects), or unknown in the case of 4 individuals who were either unobserved or who had ambiguous phenotypes. We also performed an affecteds-only analysis to allow for misspecification of the penetrance parameters, by additionally coding unaffected individuals as having unknown phenotype. Tests of linkage are robust to trait model misspecification when marker genotypes are fully known [39, 40], as is the case for the STR marker at the location with maximum evidence of linkage (see the Results section). Such tests are sufficiently powerful that they are unlikely to miss regions with positive evidence of linkage, although poor choice of parameter values can decrease the lod score [39]. The extensive genetic and allelic heterogeneity inferred from genome scans of small pedigrees [41] is unlikely to be an issue in a single unusually large pedigree with many affected subjects, so that a single-locus trait model is likely to be adequate. STR allele frequencies were estimated by direct allele counts in the pedigree. This is sufficient because although the founder couple in generation I are unobserved regarding genotype 
data, they had a sufficient number of offspring to 'force' their inferred genotypes at most markers, as verified by examination of the inheritance vectors (IVs). Single-marker STR genome scans with both models were done using the software package LINKAGE [42], and identified a candidate linkage region which was then further analyzed with multipoint methods.

After the initial single-marker linkage analyses flagged a ROI, 3 multipoint linkage analyses were performed using denser marker panels. Stage 1 (STRs only) included a denser STR panel with additional ABI markers on a subset of individuals. Stage 2 (STRs + linkage SNPs) included Stage 1 plus evenly spaced SNPs with high heterozygosity and few missing genotypes, while Stage 3 (STRs + phasing SNPs) was identical to Stage 2 except for a few SNP substitutions to maximize phasing information for particular subjects.

A mix of computational tools was used to perform multipoint linkage analyses. Meiotic map positions (in cM) for markers were obtained from the Rutgers sex-averaged Kosambi map, which used build 36 sequence positions to establish marker order [43]. To comply with implicit assumptions in the analysis methods, we converted these positions to those based on the Haldane map function. For analyses using a dense map, we selected markers that were spaced sufficiently far apart to avoid intermarker linkage disequilibrium [44], in accordance with the requirements of the LanderGreen algorithm [45]. The pedigree is too large for exact multipoint calculations with whole-chromosome marker data, so we performed all multipoint linkage analyses with a three-step approach using Markov chain Monte Carlo (MCMC). First, the program gl_auto from the MORGAN package generated sampled realizations of IVs at each marker location for entire chromosomes, conditional on all available marker information, the genetic map, and the pedigree structure [46]. This program uses MCMC sampling employing a state-of-the-art hybrid sampler [26, 47], and can accommodate large pedigrees and many markers. We carried out computations with the LMM sampler [47], using 50\% L-sampler, and sequential imputation for setup [26]. Each analysis included 1,000 burn-in iterations, sampling by scan, generated 50,000 iterations, and saved 1,000 realizations for inference. Second, we used the program IBDgraph $[46,48]$ to identify equivalence classes among the sampled IVs at each marker. Finally, we used the LINKAGE package [42] to compute likelihoods for one representative of each equivalence class at each marker position, in an application of the framework described by Sobel and Lange [49]. The lod scores were obtained by computing a weighted average over the equivalence classes, substantially reducing computation time relative to computing likelihoods for all realized IVs [50].

The same IVs produced for estimation of the lod scores were used to explore the extent of the haplotype around the putative risk locus in each stage of the multipoint analysis. A founder genome label (FGL) is a unique label for a particular founder chromosome. A realized IV can be translated into a set of FGLs describing the founder origin of realized haplotypes in the individuals of interest. At the risk locus, 8 of 11 affected individuals shared an allele IBD from the founder couple, and therefore have the same FGL at that location on the relevant (paternal or maternal) chromosome. For each of the 8 chromosomes carrying the risk allele, we calculated at each marker position the proportion of realized IVs where the FGL was the same as at the risk locus. If this proportion exceeded 95\%, we assumed the FGLs were identical, and below 5\%, we assumed non-identity. Proportions between 5 and $95 \%$ were considered ambiguous. The p-ter and q-ter boundaries of the ROI are then defined as the location closest to the risk locus where at least 1 of the 8 chromosomes definitely no longer shares an FGL with the risk locus.

\section{Exome Sequence Analysis}

Exome variants were filtered in several steps in order to reduce the number of candidate variants. Unlike typical scans for Mendelian disorders $[36,51]$, we did not filter on functional prediction, since we felt that this invoked a stronger assumption than is reasonable for a complex trait with reduced penetrance where, e.g., parents are unaffected. Instead, our major filter was positive evidence of linkage. Therefore, we eliminated from consideration all variants that fell outside of the interval defined by the Stage 3 linkage analyses described above. Variants were also eliminated if there were fewer than 10 reads at the position in any of the 5 sequenced subjects, if they failed sequencing quality metrics, such as a GATK quality score $<50$ or reference allele proportion $>0.75$ in heterozygotes, or if the set of genotypes in the 5 subjects was flagged as jointly improbable because of inconsistency with all sampled IVs [52]. We used variant allele frequencies from the HapMap CEU samples, or if these were unavailable, the 1,000 genomes EUR samples, both obtained from Ensembl (http://www. ensembl.org). We also eliminated variants that were not observed in either HapMap or 1,000 genomes, and yet were observed in the homozygous state in at least 1 sequenced subject, since this frequency distribution is implausible.

\section{Variant Imputation}

We performed analysis with the program GIGI, which uses a combination of both deterministic and probabilistic inference together with threshold-based allele calling $[53,54]$. Pedigree-based imputation relies on IVs. The IVs were obtained as for the linkage analyses above, for the Stage 3 marker panel. Conditional on realized IVs at the positions of the Stage 3 linkage markers, IVs were sampled at the positions of exome variants while including the possibility of recombination, given the meiotic map. Finally, for each sampled IV, probabilities of genotypes for all subjects are computed at the position of an exome variant, with the process repeated for all variants. These probabilities were computed jointly over pedigree members. Overall genotype and allele probability estimates for each variant were obtained by averaging over sampled IVs. Two types of thresholds were then used to call either genotypes or alleles. The default threshold required genotype probabilities of $80 \%$ for calling a genotype, and if this was not met, an allele probability of $90 \%$ for calling 1 of 2 alleles. This threshold used outside information on allele frequency, and was $97.6 \%$ accurate for called alleles in an earlier validation study [53]. The deterministic threshold required a stringent posterior probability of 99.99\% for both genotype and allele calling and therefore did not depend on allele frequency: alleles are only imputed when all sampled IVs are in agreement for a given variant and individual.

\section{Results}

The single-marker genome scan identified 2 regions with positive evidence of linkage. Support for linkage to chromosome $22\left(\operatorname{lod}_{\max }=1.81\right.$ and 2.58 for the reduced- 
Fig. 2. Linkage analyses shown relative to physical map positions for chromosome 22 for Stage 1, Stage 2, and Stage 3. Crosses show locations of each marker. Vertical dotted lines direct the reader towards boundaries of the risk haplotype shared among the 8 affected subjects inferred to carry the risk variant. Bars underneath the lod score curves represent chromosomal regions sharing the same founder genome label (FGL) as D22S683 (solid bars), not sharing this FGL (white bars), and ambiguous regarding FGL sharing (hatched bars). Stage 2 results are in black; Stage 3 results are in grey. The haplotype sharing patterns among the 8 subjects are identified by unique labels after the subject's corresponding sibship label from figure 1. Marker names are in online supplementary table 1 .

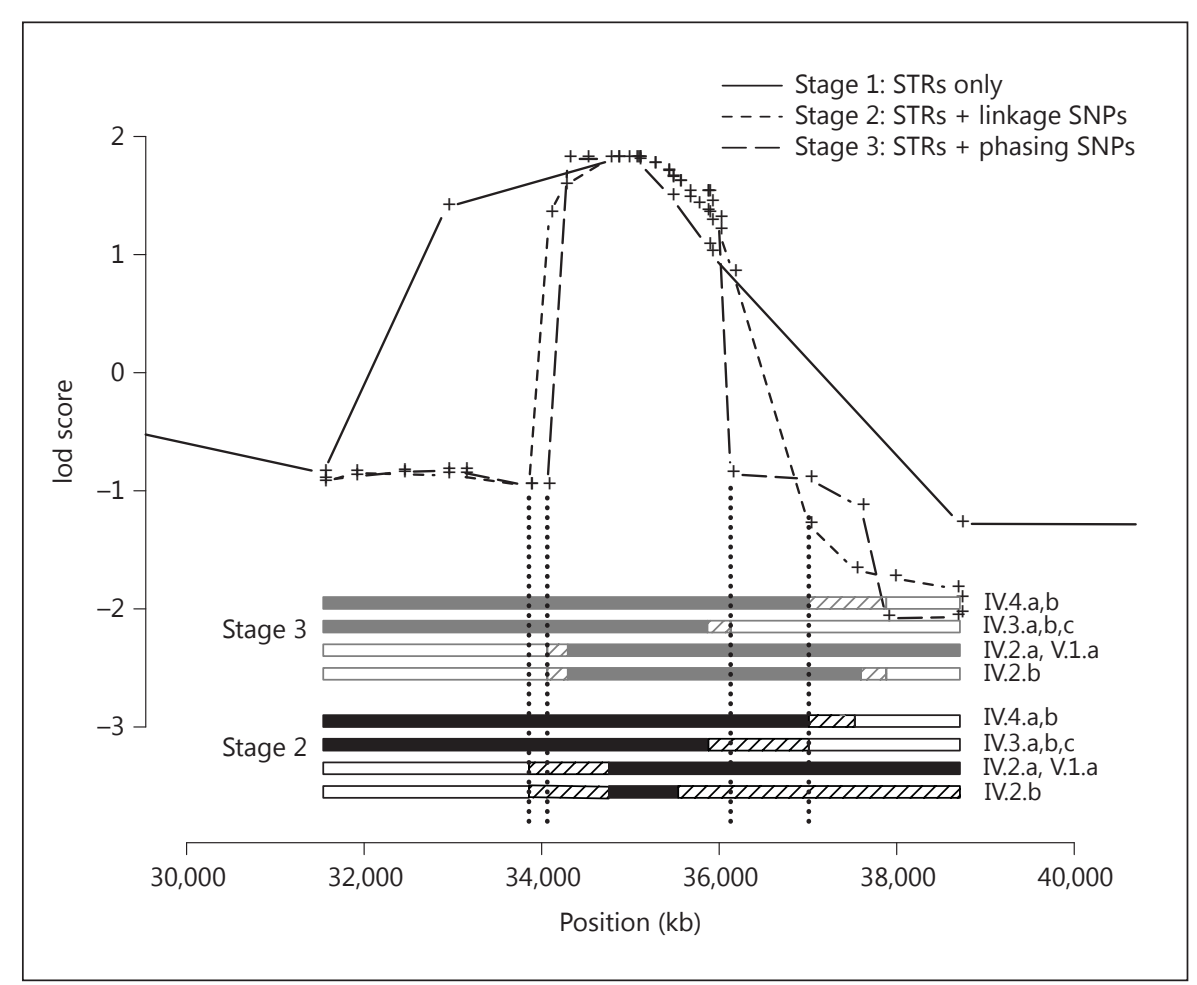

penetrance and affecteds-only analysis, respectively, at $0 \%$ recombination with $\mathrm{D} 22 \mathrm{~S} 683$ at $34.8 \mathrm{Mb}$ ) was stronger than to chromosome $6\left(\operatorname{lod}_{\max }=0.93\right.$ and 1.19 for the reduced-penetrance and affecteds-only analysis, respectively, at $10 \%$ recombination with D6S474 at $113 \mathrm{Mb}$ ). We focused subsequent analyses on the stronger signal on chromosome 22, and we limit our discussion to the results from the reduced-penetrance model because it is more conservative.

\section{Stage 1: STRs Only}

Marker density in the Stage 1 multipoint analysis was about 1 STR per $3.7 \mathrm{cM}$. Online supplementary table 1 (for all online supplementary material, see www. karger.com/doi/10.1159/000346560) and figure 2 show the multipoint reduced-penetrance lod scores at each marker location in Stages 1, 2 and 3. The multipoint lod score maximized at 1.81 at the position of D22S683, matching the single-marker analysis result because D22S683 is fully informative in this pedigree. Eight out of the 11 affected individuals share the same allele IBD (the tagging allele) from the original founders at D22S683, while 3 additional affected subjects across 3 sibships all share a distinct allele that originates in a founder who married into the family in generation II. Online supple- mentary table 2 provides the FGL sharing probabilities at each marker for the 8 individuals carrying the tagging allele. These probabilities identify a ROI with boundaries that correspond to the positions of markers with negative lod scores, and results in a 7.2-Mb region extending q-ter of D22S280 to p-ter of D22S423.

\section{Stage 2: STRs + Linkage SNPs}

The combined STR and linkage SNP panel results in an average marker density of about 1 marker per 0.5 cM. As shown in figure 2 and online supplementary table 1, the Stage 2 multipoint maximum lod score at D22S683 remains unchanged. However, the lod score at D22S685 - the next marker in the Stage 1 analysis p-ter to D22S683 - drops from 1.4 in Stage 1 to -0.83 in Stage 2 because neighboring SNPs clearly indicate that D22S685 does not co-segregate with D22S683 in the 8 affected individuals sharing the tagging allele. Bars underneath the lod score curves in figure 2 represent chromosomal regions sharing the same FGL as D22S683 (solid bars), not sharing this FGL (white bars), and ambiguous regarding FGL sharing (hatched bars). The p-ter boundary of the risk haplotype surrounding D22S683 is more precisely localized between rs134215 and rs1076139 by subjects IV.2.a, V.1.a, and IV.2.b (fig. 2; online suppl. table 1). 
Table 1. Mean fraction of alleles per genotype called by imputation

\begin{tabular}{|c|c|c|c|c|}
\hline \multirow[t]{2}{*}{ Subject group } & \multirow{2}{*}{$\begin{array}{l}\text { Unob- } \\
\text { served } \\
\text { subjects }\end{array}$} & \multirow{2}{*}{$\begin{array}{l}\text { Variant } \\
\text { type }\end{array}$} & \multicolumn{2}{|c|}{ Calling procedure } \\
\hline & & & default & $\begin{array}{l}\text { deter- } \\
\text { ministic }\end{array}$ \\
\hline Whole pedigree & $42^{\mathrm{a}}$ & All & 0.698 & 0.377 \\
\hline Whole pedigree & 38 & All & 0.718 & 0.417 \\
\hline Whole pedigree & $42^{\mathrm{a}}$ & Rare & 0.956 & 0.391 \\
\hline Whole pedigree & 38 & Rare & 0.951 & 0.431 \\
\hline Sibs ${ }^{\mathrm{b}}$ & 11 & All & 0.793 & 0.498 \\
\hline Parents, avuncular ${ }^{\mathrm{b}}$ & 8 & All & 0.772 & 0.539 \\
\hline $\begin{array}{l}\text { Grandparent, } \\
\text { great-avuncular }{ }^{\mathrm{b}}\end{array}$ & 6 & All & 0.685 & 0.371 \\
\hline
\end{tabular}

${ }^{a}$ Includes some subjects without imputation potential - who can share no allele(s) IBD with a sequenced subject.

${ }^{\mathrm{b}}$ Relative to an individual at the bottom of the pedigree who has sequence data.

Similarly, the q-ter boundary of the risk haplotype is more precisely located between rs133710 and rs575076 by subjects IV.3.a, IV.3.b, and IV.3.c. Thus, the Stage 2 analysis narrowed the ROI by a factor of $>50 \%$ to a $3.15-\mathrm{Mb}$ region between rs134215 and rs5750576.

\section{Stage 3: STRs + Phasing SNPs}

Multipoint linkage analysis on Stage 3 markers further narrowed the linkage region and risk haplotype. The p-ter boundary of the risk haplotype lies between rs138794 and rs5750116 (subjects IV.2.a, V.1.a, and IV.2.b), and the qter boundary lies between D22S1045 and rs4328731 (subjects IV.3.a, IV.3.b, and IV.3.c; fig. 2; online suppl. table 1). The Stage 3 analyses further narrowed the ROI by an additional $34 \%$ to $2.1 \mathrm{Mb}$, between rs138794 and rs4328731. In conclusion, the linkage analyses provide a 2.1-Mb ROI on a haplotype which is carried by 8 of the 11 affected individuals.

\section{Selection of Individuals for Exome Sequencing}

Budgetary considerations allowed the selection of 5 subjects for exome sequencing. Of the 5 subjects chosen for exome sequencing, 4 were affected subjects from separate nuclear families. Together, 3 of these subjects (from sibships V.1, IV.3, and IV.5; fig. 1) represented both major branches of the pedigree, and carry the risk haplotype. The 4th affected subject (from sibship IV.5) did not carry this haplotype. The 5th subject was an unaffected individual (II.1.b) who had multiple affected descendants, and was the grandparent of 3 of the 4 affected subjects chosen for sequencing. This individual was as close to the founder couple as possible and was chosen to facilitate the imputation of variants into his/her descendants [55].

\section{Exome Sequence Results}

Before filtering, exome sequencing provided 27,48428,133 variants per subject genome-wide, only 80 of which were in the Stage 3 ROI. Of these 80, 9 were removed by the GATK filter, and a further 3 due to low read depth. Online supplementary table 3 shows the remaining 68 variants, their frequencies in reference populations, and observed counts in each of the 5 exomed individuals. Of these $68,5.9 \%$ were rare, defined as a minor allele frequency $<0.05$, and 2 were novel. An additional 79 variants in the flanking regions of the ROI were also used to provide larger sample sizes for imputation metrics.

\section{Imputation Results}

Overall imputation rates were high (table 1). Across the entire pedigree, $37.7 \%$ of the unobserved alleles for the 147 exome variants were called by deterministic imputation, with this number increasing to $69.8 \%$ when the default threshold was used. Sequencing a small subset of the pedigree necessarily limited the number of independent founder chromosomes that could be represented in the sequence data: our selection of subjects used to generate exome data left 2 of the 11 founder genomes completely unrepresented. In addition, non-founders other than full-siblings share at most 1 allele IBD with 1 of the sequenced individuals, limiting the number of alleles that can be imputed from the exome data. We therefore report imputation results both for subjects who could share an allele IBD with one of the sequenced individuals and relative to all pedigree members. Restricting the summary to the 38 subjects who could share an allele IBD with a sequenced subject gave a higher call rate of $41.7 \%$ under the deterministic threshold, or $71.8 \%$ under the default threshold. The deterministic call rate of $41.7 \%$ is $83 \%$ of the maximum achievable (50\%) for unilineal relatives who share an IBD segment. As expected, close relatives, including siblings, parents and avuncular relatives, had the highest imputation rates: $77.2-79.3 \%$ for the default threshold, and 49.8-53.9\% with the deterministic threshold. However, even individuals related as grandparent or great-avuncular had similar imputation rates overall, indicating that the haplotype information propagates efficiently through the pedigree when guided by good framework marker data.

Imputation rates depended on choice of call procedure and the allele frequency of the variant being called. As 
Fig. 3. Number of imputed exome variant alleles under the default threshold for all 147 variant sites (a) and for the variants with rare minor allele frequencies $(\mathbf{b})$.
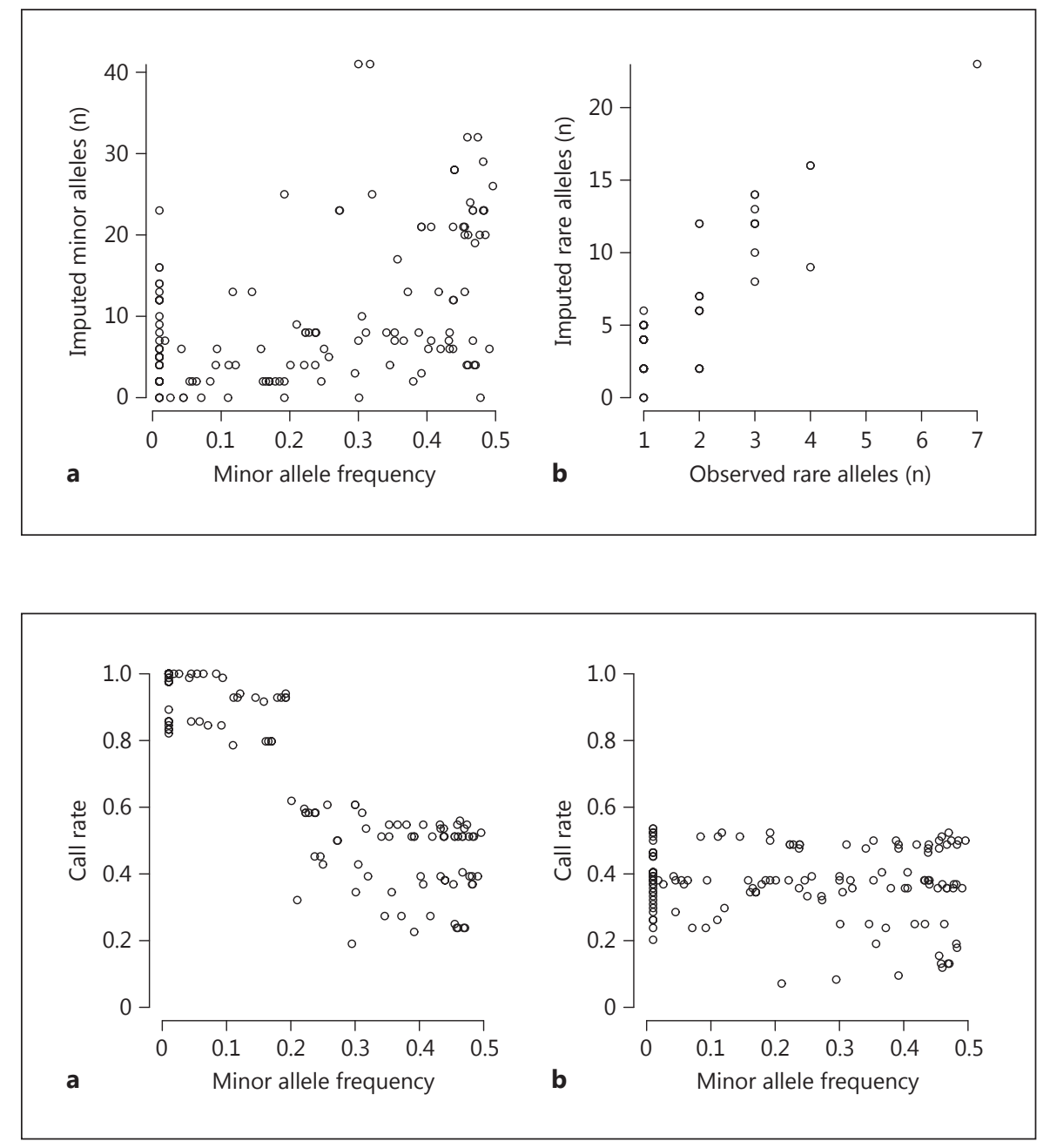

Fig. 4. Probability of calling an exome variant allele as a function of minor allele frequency for the 147 variant sites based on the default threshold (a) and on the deterministic threshold (b). genotypes with the major allele in the population, which assists with phasing [56]. With deterministic calling (fig. 4b), this effect of allele frequency was no longer evident, revealing the ability of IV information to impute variants while remaining agnostic to population allele frequencies.

Call rates increased with the inclusion of one key unaffected subject (fig. 5). This subject (II.1.b in fig. 1) carried the risk haplotype and had many descendants, including 3 grandchildren with exome data, 2 of whom carry the risk haplotype. Inclusion of sequence data from II.1.b lead to increased call rates for both the default (fig. 5a) and the deterministic thresholds (fig. 5b). Some of the individuals with the greatest gains in fraction of alleles imputed (fig. 5b), from 0.085 to 0.412 with deterministic imputation, were siblings of the one affected individual with exome data who did not carry the risk haplotype. SNP 
Fig. 5. Exome variant call rates in the 42 subjects with no direct exome genotyping when exome data for only the 4 subjects at the bottom of the pedigree are used versus when exome data for all 5 subjects are used, for the default threshold (a) and for the deterministic threshold (b).

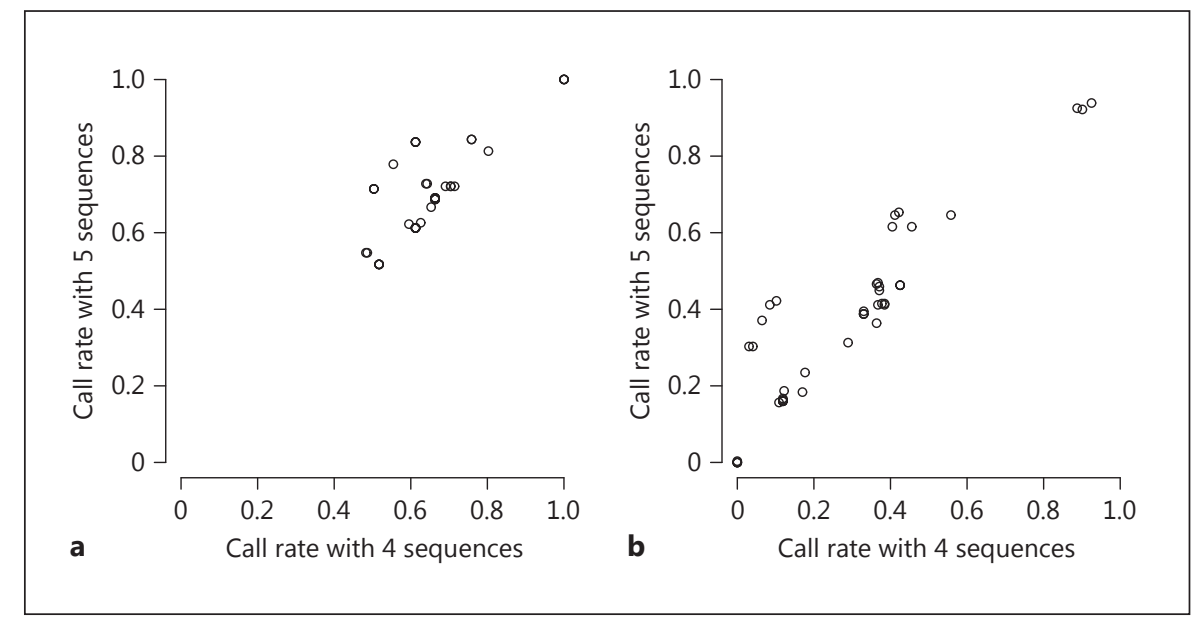

genotyping failed in this affected individual. Therefore, because at least 2 directly genotyped copies of at least 1 haplotype are required for pedigree-based phasing [56], removal of the shared haplotype in the grandparent (II.1.b) eliminated most of the information needed to phase the exome variants relative to the SNPs, and thus to permit imputation of variant alleles into the siblings.

\section{Implicated Imputed Variants}

One of the 68 exome variants identified within the ROI was both present in all 4 subjects carrying the risk haplotype and also rare (table 2). None of the 3 additional rare variants in the ROI were compatible with being a risk variant since they were not present in all 4 subjects who carried the risk haplotype. We also consider, primarily to illustrate the properties of our imputation method, a variant that is shared among the 4 carriers of the risk haplotype but is more common (rs7290488). There were also approximately 30 variants with more common allele frequencies that were shared among the 4 sequenced risk haplotype carriers, but unlike the risk haplotype had multiple founder origins in the pedigree, as indicated by their IVs. These common variants are not discussed further because they are not consistent with the rare-variant hypothesis for the disease.

A rare variant in MYH9 (myosin heavy chain 9) at $36,689,462$ bp (build 37) was the most plausible candidate (table 2; online suppl. table 3 ) given 2 criteria. First, this variant is very rare and it has been reported only once in outside databases. The variant allele was in the heterozygous state in all 4 carriers of the risk haplotype, and was not observed in the one affected subject without the risk haplotype. Second, the number of variant alleles imputed was greater than for all 3 other rare variants in the ROI. This variant allele segregated perfectly with the risk haplotype, as inferred by a large increase in the number of variant alleles called by imputation, increasing from the observed 4 alleles in the sequenced subjects to 20 alleles in the rest of the pedigree under the default threshold. These 20 alleles accounted for all subjects who inherited the risk haplotype. However, probably because there were no homozygotes to help with phasing for the variant alleles, deterministic calling added only 4 additional copies of the variant allele. Finally, imputation of this variant was insensitive to exclusion of the exome data for the unaffected grandparent (II.1.b), which resulted in the loss of only 1 imputed allele. This suggests that the 4 observed exomes that represent the risk haplotype are more than adequate to phase the MYH9 variant.

To illustrate the properties of our imputation method, we also consider rs7290488 in the gene C1QTNF6 (C1q and tumor necrosis factor-related protein 6). The frequency of the variant allele, at approximately 0.2 in the HapMap CEU sample, is considerably higher than expected under the rare-variant hypothesis. The number of imputed copies of the variant allele was 25 or 23 using default or deterministic thresholds, respectively. It is likely that this high deterministic call rate was the consequence of homozygosity for the variant allele in II.1.b. Unlike the MYH9 variant, imputation of this variant allele was sensitive to inclusion of the grandparental exome data. Exclusion of II.1.b's exome data caused a drop of in the number of imputed copies from 23 to 3 (deterministic calling) and from 25 to 15 (default calling), indicating that the affected subjects alone do not provide adequate phase information regarding this variant. This variant is unlike- 
Table 2. Imputation results in 2 variants in the Stage 3 ROI on chromosome 22

\begin{tabular}{|c|c|c|c|c|c|c|c|c|c|}
\hline \multirow[t]{2}{*}{ SNP } & \multirow[t]{2}{*}{$\begin{array}{l}\text { Position (bp) } \\
\text { HG19 }\end{array}$} & \multicolumn{2}{|l|}{ Base-pair } & \multirow[t]{2}{*}{$\begin{array}{l}\text { Variant } \\
\text { frequency }\end{array}$} & \multirow{2}{*}{$\begin{array}{l}\text { Variants } \\
\text { observed } \\
\mathrm{n}\end{array}$} & \multicolumn{2}{|c|}{$\begin{array}{l}\text { Variants imputed, } \mathrm{n} \\
\text { ( } 5 \text { exomes: } 4 \text { exomes) }\end{array}$} & \multirow[t]{2}{*}{ Gene } & \multirow[t]{2}{*}{$\begin{array}{l}\text { Prediction } \\
\text { (polyphen) }\end{array}$} \\
\hline & & reference & variant & & & default & deterministic & & \\
\hline NA & $36,689,462$ & G & A & NA & 4 & $16: 15$ & $4: 3$ & MYH9 & Coding synonymous \\
\hline rs7290488 & $37,581,383$ & $\mathrm{C}$ & $\mathrm{T}$ & 0.19 & 5 & $25: 15$ & $23: 3$ & C1QTNF6 & Benign missense \\
\hline
\end{tabular}

${ }^{a}$ Variant frequency in the HapMap CEU sample.

ly to be a risk variant, both because of its relatively high frequency, and also because the total number of copies imputed into the pedigree exceeds the number of observed copies of the risk haplotype. Thus, this variant allele was inferred to exist in 6 additional subjects who did not have autism.

\section{Discussion}

We have illustrated both some of the possibilities and the complications of analyzing sequence data in a large pedigree, with the goal of identifying risk alleles contributing to a complex trait with reduced penetrance. The large autism pedigree analyzed here is highly unusual in its size and number of generations represented, stimulating an analysis under the assumption that segregating rare genetic variation underlies the trait. By using tools that allow analysis of dense marker data on intact, large pedigrees, we were able to obtain strong evidence for localization of a risk locus to chromosome 22, precisely bound the interval likely to carry the risk variant, and prioritize evaluation of all exome sequence variants within that region. Knowledge of carrier status for the risk haplotype was used to select subjects for sequencing. These procedures, coupled with additional constraints that assume a rare variant allele frequency, reduced the number of candidate exome sequence variants for autism risk in this pedigree to one. A second, weaker, candidate variant is also possible, depending on prior expectation for how rare the frequency of a causal variant must be along with greater tolerance for reduced penetrance of the trait. Although these 2 candidate variants would have been identified by sequencing only the 3 affected carriers, we would have been unable to exclude many other variants without also sequencing the grandparent and affected non-carrier of the risk haplotype.

Rare Variants in Autism
Any conclusion about causal or risk variants in the implicated region of chromosome 22 requires accepting a reduced-penetrance model with some sporadic cases, even in this large pedigree. The stronger linkage signal under the affecteds-only analysis suggests that our reduced-penetrance model overestimated the penetrance of the possible risk allele driving the linkage signal on chromosome 22. More than one contribution to the diagnosis is required, since there are 3 affected males in 3 different nuclear families who are affected, yet did not inherit the risk haplotype. Reduced penetrance of the risk allele(s) is a requirement for the transmission of a risk allele through apparently normal parents, and also to explain discrepancies between the inherited risk alleles and the phenotypes of individuals who are at risk: those who inherited a risk allele yet are unaffected. There are 3 such unaffected females who share both the MYH9 and C1QTNF6 variants, and 3 additional such unaffected females who share the C1QTNF6 variant. A lower penetrance in females than males is expected, given the approximately 3.8:1 ratio of males:females with autism [57], but there are fewer such discrepancies required for the MYH9 than the C1QTNF6 variant.

Our results show that additional genotype data provides information that is useful for pursuing risk variants, but only up to a point. In this data set, the denser SNP marker data provided equal, but not more, support for a risk locus compared to the information provided by the initial sparse STR markers. However, SNP markers did add considerable precision for determining the boundaries of the risk haplotype, leading to a sharp reduction in the number of variants in the exome data that were of interest. Imputation of alleles from the exome data in the context of the denser marker data then provided a rapid and inexpensive approach for determining which of the variants were most consistent with the inheritance of the risk haplotype, and provided, at no additional laboratory 
cost, an additional 1.5-2.7 times the number of alleles than were directly measured in the exome data, depending on the genotype call threshold used. This suggests the need for future investigation into tradeoffs between direct genotyping versus imputation in the search for causal variants in pedigrees.

Although our analysis identified 2 variants in the exome data that are consistent with inheritance of the putative underlying trait locus, neither variant is compelling as a causal variant for autism, given current biological knowledge. The variant in MYH9 is most consistent with both the observed inheritance pattern and with the rarevariant hypothesis. However, the variant seen in this family is not predicted to have a functional effect. Also, mutations in MYH9 have only been reported to be associated with abnormal platelets, nephritis, and deafness (OMIM: 160775), although it has been suggested that platelet defects could be involved in diseases such as autism and schizophrenia [58]. The second gene, C1QTNF6, similarly has little published support that would suggest a role in autism, and also is less compelling, given the relatively high frequency of the variant allele and greater number of inferred unaffected carriers in the pedigree. Of course, it is possible that different alleles in either of these genes could have very different phenotypic effects than previously associated with earlier noted mutations, and that either of these identified variants is, in fact, a cause of autism in this pedigree.
It is also possible that the causal variant for autism risk is not in the exome sequence data and that we have therefore not seen it yet. The focus on transcribed DNA that is typical in human genetics may miss important variants that affect quantitative, rather than qualitative, risk [59]. In the search for the genetic basis of a complex trait, such as autism, it may be necessary to sequence non-coding DNA to determine if there are other variants of equal or greater interest. One advantage of analysis of a large pedigree with linkage analysis results, such as we have here, is that this information is useful for prioritizing the many sequence variants yet to be identified in non-coding DNA: the variants of interest are likely to be in the regions implicated by positive linkage evidence, which strongly limits the search space. Further restriction can be obtained with filters based on allele frequency and with the imputation approach used here, which provides information about co-segregation with the trait in a computationally rapid manner. Creative approaches such as these will be necessary to make headway in understanding the biology of complex traits.

\section{Acknowledgements}

This study was supported by funding from the National Institutes of Health, grants GM046255, MH094293, MH094400, HD055782, MH092367, HG005608, and AG040184.

\section{References}

1 Morton NE: Sequential tests for the detection of linkage. Am J Hum Genet 1955;7:277-318.

-2 Elston RC, Stewart J: A general model for the genetic analysis of pedigree data. Hum Hered 1971;21:523-542.

3 Ott J: Estimation of the recombination fraction in human pedigrees: efficient computation of the likelihood for human linkage studies. Am J Hum Genet 1974;26:588-597.

4 Botstein D, White RL, Skolnick M, Davis RW: Construction of a genetic linkage map in man using restriction fragment length polymorphisms. Am J Hum Genet 1980;32:314-331.

5 Gusella JF, Wexler NS, Conneally PM, Naylor SL, Anderson MA, Tanzi RE, Watkins PC, Ottina K, Wallace MR, Sakaguchi AY, Young $\mathrm{AB}$, Shoulson I, Bonilla E, Martin JB: A polymorphic DNA marker genetically linked to Huntington's disease. Nature 1983;306:234238.

6 Amberger J, Bocchini C, Hamosh A: A new face and new challenges for Online Mendelian Inheritance in Man $\left(\mathrm{OMIM}^{\circ}\right)$. Hum $\mathrm{Mu}$ tat 2011;32:564-567.
7 Hall JM, Lee MK, Newman B, Morrow JE, Anderson LA, Huey B, King MC: Linkage of early-onset familial breast cancer to chromosome 17q21. Science 1990;250:1684-1689.

8 Goate A, Chartier-Harlin MC, Mullan M, Brown J, Crawford F, Fidani L, Giuffra L, Haynes A, Irving N, James L, Mant R, Newton P, Rooke K, Roques P, Talbot C, PericakVance M, Roses A, Williamson R, Rossor M, Owen M, Hardy J: Segregation of a missense mutation in the amyloid precursor protein gene with familial Alzheimer's disease. Nature 1991;349:704-706.

-9 Schellenberg GD, Bird TD, Wijsman EM, Orr HT, Anderson L, Nemens E, White JA, Bonnycastle L, Weber JL, Alonso ME, et al: Genetic linkage evidence for a familial Alzheimer's disease locus on chromosome 14. Science 1992;258:668-671.

10 Risch N, Merikangas KR: The future of genetic studies of complex human diseases. Science 1996;273:1516-1517.
1 Collins FS, Guyer MS, Chakravarti A: Variations on a theme: cataloging human DNA sequence variation. Science 1997;278:15801581.

12 McClellan J, King MC: Genetic heterogeneity in human disease. Cell 2010;141:210-217.

$\checkmark 13$ Hindorff LA, Sethupathy P, Junkins HA, Ramos EM, Mehta JP, Collins FS, Manolio TA: Potential etiologic and functional implications of genome-wide association loci for human diseases and traits. Proc Natl Acad Sci USA 2009;106:9362-9367.

14 Manolio TA, Collins FS, Cox NJ, Goldstein DB, Hindorff LA, Hunter DJ, McCarthy MI, Ramos EM, Cardon LR, Chakravarti A, Cho $\mathrm{JH}$, Guttmacher AE, Kong A, Kruglyak L, Mardis E, Rotimi CN, Slatkin M, Valle D, Whittemore AS, Boehnke M, Clark AG, Eichler EE, Gibson G, Haines JL, Mackay TFC, McCarroll SA, Visscher PM: Finding the missing heritability of complex diseases. Nature 2009;461:747-753. 


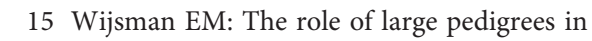
an era of high-throughput sequencing. Hum Genet 2012;131:1555-1563.

16 Bailey-Wilson JE, Wilson AF: Linkage analysis in the next-generation sequencing era. Hum Hered 2011;72:228-236.

17 Wijsman EM, Amos CI: Genetic analysis of simulated oligogenic traits in nuclear and extended pedigrees: summary of GAW10 contributions. Genet Epidemiol 1997;14:719735 .

18 Hinrichs AL, Suarez BK: Incorporating linkage information into a common disease/rare variant framework. Genet Epidemiol 2011; 35:S74-S79.

$\checkmark 19$ Wilson AF, Ziegler A: Lessons learned from genetic analysis workshop 17: transitioning from genome-wide association studies to whole-genome statistical genetic analysis. Genet Epidemiol 2011;35:S107-S114.

20 Rosenthal EA, Ronald J, Rothstein J, Rajagopalan R, Ranchalis J, Wolfbauer G, Albers JJ, Brunzell JD, Motulsky AG, Rieder MJ, Nickerson DA, Wijsman EM, Jarvik GP: Linkage and association of phospholipid transfer protein activity to LASS4. J Lipid Res 2011;52: 1837-1846.

21 Musunuru K, Pirruccello JP, Do R, Peloso GM, Guiducci C, Sougnez C, Garimella KV, Fisher S, Abreu J, Barry AJ, Fennell T, Banks E, Ambrogio L, Cibulskis K, Kernytsky A, Gonzalez E, Rudzicz N, Engert JC, DePristo MA, Daly MJ, Cohen JC, Hobbs HH, Altshuler D, Schonfeld G, Gabriel SB, Yue P, Kathiresan S: Exome sequencing, ANGPRL3 mutations, and familial combined hypolipidemia. N Engl J Med 2010;363:2220-2227.

-22 Smith KR, Bromhead CJ, Hildebrand MS, Shearer AE, Lockhart PJ, Najmabadi H, Leventer RJ, McGillivray G, Amor DJ, Smith RJ, Bahlo M: Reducing the exome search space for mendelian diseases using genetic linkage analysis of exome genotypes. Genome Biol 2011;12:R85.

23 Ng SB, Nickerson DA, Bamshad MJ, Shendure J: Massively parallel sequencing and rare disease. Hum Mol Genet 2010;19:R119R124.

-24 Ng SB, Turner EH, Robertson PD, Flygare SD, Bigham AW, Lee C, Shaffer T, Wong M, Bhattacharjee A, Eichler EE, Bamshad M, Nickerson DA, Shendure J: Targeted capture and massively parallel sequencing of 12 human exomes. Nature 2009;461:272-276.

-25 Roach JC, Glusman G, Smit AFA, Huff CD, Hubley R, Shannon PT, Rowen L, Pant KP, Goodman N, Bamshad M, Shendure J, Drmanac R, Jorde LB, Hood L, Galas DJ: Analysis of genetic inheritance in a family quartet by whole-genome sequencing. Science 2010;328:636-639.

-26 Wijsman EM, Rothstein JH, Thompson EA: Multipoint linkage analysis with many multiallelic or dense diallelic markers: Markov chain-Monte Carlo provides practical approaches for genome scans on general pedigrees. Am J Hum Genet 2006;79:846-858.
27 Schellenberg GD, Dawson G, Sung YJ, Estes A, Munson J, Rosenthal E, Rothstein J, Flodman P, Smith M, Coon H, Leong L, Yu CE, Stodgell C, Rodier PM, Spence MA, Minshew N, McMahon WM, Wijsman EM: Evidence for multiple loci from a genome scan of autism kindreds. Mol Psychiatry 2006;11:10491060.

28 Coon H, Matsunami N, Stevens J, Miller J, Pingree C, Camp NJ, Thomas A, Krasny L, Lainhart J, Leppert MF, McMahon W: Evidence for linkage on chromosome $3 q 25-27$ in a large autism extended pedigree. Hum Hered 2005; 60:220-226.

29 Coon H, Matsunami N, Stevens J, Miller J, Pingree C, Camp NJ, Thomas A, Krasny L, Lainhart J, Leppert MF, McMahan W: Evidence for linkage on chromosome 3q25-27 in a large autism extended pedigree. Hum Hered 2006;60:220-226.

30 Allen-Brady K, Miller J, Matsunami N, Stevens J, Block H, Farley M, Krasny L, Pingree C, Lainhart J, Leppert M, McMahon WM, Coon H: A high-density SNP genome-wide linkage scan in a large autism extended pedigree. Mol Psychiatry 2009;14:590-600.

-31 Allen-Brady K, Robison R, Cannon D, Varvil T, Villalobos M, Pingree C, Leppert MF, Miller J, McMahon WM, Coon H: Genome-wide linkage in Utah autism pedigrees. Mol Psychiatry 2010;15:1006-1015.

32 Hallmayer J, Spiker D, Lotspeich L, McMahon WM, Petersen PB, Nicholas P, Pingree C, Ciaranello RD: Male-to-male transmission in extended pedigrees with multiple cases of autism. Am J Med Genet 1996;67:13-18.

-33 Ma DQ, Cuccaro ML, Jaworski JM, Haynes CS, Stephan DA, Parod J, Abramson RK, Wright HH, Gilbert JR, Haines JL, PericakVance MA: Dissecting the locus heterogeneity of autism: significant linkage to chromosome 12q14. Mol Psychiatry 2007;12:376384.

34 Kilpinen H, Ylisaukko-Oja T, Rehnstrom K, Gaal E, Turunen JA, Kempas E, von Wendt L, Varilo T, Peltonen L: Linkage and linkage disequilibrium scan for autism loci in an extended pedigree from Finland. Hum Mol Genet 2009;18:2912-2921.

35 Brkanac Z, Spencer D, Shendure J, Robertson PD, Matsushita M, Vu T, Bird TD, Olson MV, Raskind WH: IFRD1 is a candidate gene for SMNA on chromosome 7q22-q23. Am J Hum Genet 2009;84:692-697.

36 Ng SB, Buckingham KJ, Lee C, Bigham AW, Tabor HK, Dent KM, Huff CD, Shannon PT, Jabs EW, Nickerson DA, Shendure J, Bamshad MJ: Exome sequencing identifies the cause of a mendelian disorder. Nat Genet 2010;42:30-35.
37 O’Roak BJ, Vives L, Girirajan S, Karakoc E, Krumm N, Coe BP, Levy R, Ko A, Lee C, Smith JD, Turner EH, Stanaway IB, Vernot B, Malig M, Baker C, Reilly B, Akey JM, Borenstein E, Rieder MJ, Nickerson DA, Bernier R, Shendure J, Eichler EE: Sporadic autism exomes reveal a highly interconnected protein network of de novo mutations. Nature 2012;485:246-250.

38 Laurie CC, Doheny KF, Mirel DB, Pugh EW, Bierut LJ, Bhangale T, Boehm F, Caporaso NE, Cornelis MC, Edenberg HJ, Gabriel SB, Harris EL, Hu FB, Jacobs KB, Kraft P, Landi MT, Lumley T, Manolio TA, McHugh C, Painter I, Paschall J, Rice JP, Rice KM, Zheng XW, Weir BS, GENEVA Investigators: Quality control and quality assurance in genotypic data for genome-wide association studies. Genet Epidemiol 2010;34:591-602.

-39 Clerget-Darpoux F, Bonaiti-Pellie C, Hochez $\mathrm{J}$ : Effects of misspecifying genetic parameters in lod score analysis. Biometrics 1986;42:393399.

40 Williamson JA, Amos CI: Guess lod approach: sufficient conditions for robustness. Genet Epidemiol 1995;12:163-176.

41 Devlin B, Scherer SW: Genetic architecture in autism spectrum disorder. Curr Opin Genet Dev 2012;22:229-237.

42 Lathrop GM, Lalouel JM, Julier C, Ott J: Strategies for multilocus linkage analysis in humans. Proc Natl Acad Sci USA 1984;81:34433446.

43 Matise TC, Chen F, Chen WW, De la Vega FM, Hansen M, He CS, Hyland FCL, Kennedy GC, Kong XY, Murray SS, Ziegle JS, Stewart WCL, Buyske S: A second-generation combined linkage-physical map of the human genome. Genome Res 2007;17:1783-1786.

44 Wilcox MA, Pugh EW, Zhang H, Zhong X, Levinson DF, Kennedy GC, Wijsman EM: Comparison of single-nucleotide polymorphisms and microsatellite markers for linkage analysis in the COGA and simulated data sets for Genetic Analysis Workshop 14: Presentation Groups 1, 2, and 3. Genet Epidemiol 2005;29(suppl 1):S7-S28.

45 Lander ES, Green PJ: Construction of multilocus genetic maps in humans. Proc Natl Acad Sci USA 1987;84:2363-2367.

46 Thompson EA: The structure of genetic linkage data: from LIPED to $1 \mathrm{M}$ SNPs. Hum Hered 2011;71:86-96.

47 Tong LP, Thompson E: Multilocus lod scores in large pedigrees: combination of exact and approximate calculations. Hum Hered 2008; 65:142-153.

48 Koepke H, Thompson EA: Efficient testing operations on dynamic graph structures using strong hash functions: Department of Statistics, technical reports. Seattle, University of Washington, 2010.

-49 Sobel E, Lange K: Descent graphs in pedigree analysis: applications to haplotyping, location scores, and marker-sharing statistics. Am J Hum Genet 1996;58:1323-1337. 
50 Marchani EE, Wijsman EM: Estimation and visualization of identity-by-descent within pedigrees simplifies interpretation of complex trait analysis. Hum Hered 2011;72:289297.

51 Ng SB, Bigham AW, Buckingham KJ, Hannibal MC, McMillin MJ, Gildersleeve HI, Beck AE, Tabor HK, Cooper GM, Mefford HC, Lee C, Turner EH, Smith JD, Rieder MJ, Yoshiura K, Matsumoto N, Ohta T, Niikawa N, Nickerson DA, Bamshad MJ, Shendure J: Exome sequencing identifies MLL2 mutations as a cause of Kabuki syndrome. Nat Genet 2010; 42:790-793.

52 Wijsman EM, Cheung CYK, Thompson EA: Detection of genotyping errors in dense markers on large pedigrees. Genet Epidemiol 2012;36:126.
53 Cheung CYK, Thompson EA, Wijsman EM: In silico genotype imputation on large pedigrees. Genet Epidemiol 2010;34:919.

54 Cheung CYK, Thompson EA, Wijsman EM: GIGI: an effective approach to effective imputation of genotypes in large pedigrees. Am Hum Genet 2012; submitted.

55 Burdick JT, Chen WM, Abecasis GR, Cheung VG: In silico method for inferring genotypes in pedigrees. Nat Genet 2006;38:1002-1004.

56 Wijsman EM: A deductive method of haplotype analysis in pedigrees. Am J Hum Genet 1987;41:356-373.

57 Fombonne E: The epidemiology of autism: a review. Psychol Med 1999;29:769-786.
Freson K, Labarque V, Thys C, Wittevrongel C, Van Geet C: What's new in using platelet research? To unravel thrombopathies and other human disorders. Eur J Pediatr 2007; 166:1203-1210.

59 Webster JA, Gibbs JR, Clarke J, Ray M, Zhang WX, Holmans P, Rohrer K, Zhao A, Marlowe L, Kaleem M, McCorquodale DS, Cuello C, Leung D, Bryden L, Nath P, Zismann VL, Joshipura K, Huentelman MJ, Hu-Lince D, Coon KD, Craig DW, Pearson JV, Heward CB, Reiman EM, Stephan D, Hardy J, Myers AJ: Genetic control of human brain transcript expression in Alzheimer disease. Am J Hum Genet 2009;84:445-458. 